[pp.73-80]

Vol. 4(1), Enero - Junio, 2016

http://dx.doi.org/10.15359/rnh.4-1.4

\title{
La vejez y el cerebro
}

\section{Old Age and the Brain}

Victoria I. Tirro

Universidad Central de Venezuela

Venezuela

victoriatirro@gmail.com

\section{Resumen}

El paso de los años lleva consigo una variedad de cambios que generan ganancias y pérdidas de diversa índole; por lo que el envejecer no puede ser visto sólo como una etapa de decadencia. Resulta importante, pues, exponer mitos y realidades con relación al cerebro, la memoria y la capacidad de seguir siendo activo. Para entender estos aspectos, las investigaciones en el ámbito de las neurociencias del siglo XXI han sido un excelente aporte.

Palabras clave: vejez, cerebro, memoria, hipocampo, neurociencias

\begin{abstract}
As the years pass by, changes come with them generating profits and losses of all kind. So, aging cannot be considered as just a decadence stage. Therefore, it is important to present myths and realities related to the brain, memory and capacity of keeping active. To understand these aspects, neuroscience researches in the $21 \mathrm{st}$ century have been an excellent contribution.
\end{abstract}

Keywords: Old age, brain, memory, hippocampus, neurosciences.

Todo lo que no se usa, se atrofia”, así reza un viejo refrán que refleja, a cabalidad, la realidad del envejecimiento y el deterioro cognitivo; es, además, el principio por el cual se rige una nueva visión de la vejez. 


\section{Cambiando paradigmas}

El estudio del cerebro estuvo bastante tiempo signado por la creencia de que el sistema nervioso central poseía una estructura inmutable e irreparable tanto funcional como anatómicamente, lo que le otorgaba el carácter de un sistema terminado y definitivo, sometido a un proceso de desgaste e involución con el paso de los años, sin posibilidades de reparación ante alguna lesión.

Gracias a los actuales avances científicos, la inflexibilidad del cerebro como paradigma central se ha visto derrocado, al considerarse la existencia de un mecanismo plástico, donde la actividad en la cual se somete la persona ocasiona cambios en la neuroquímica y la neuroanatomía cerebral, produciéndose un fenómeno de moldeaje y reorganización, en donde se fortalecen o establecen nuevas conexiones sinápticas y se debilitan aquellas que no son frecuentemente utilizadas. En la actualidad dichas estimulaciones parecieran inducir el proceso de generación neuronal.

La nueva percepción de la vejez como fenómeno individual y social portador de una serie de aspectos positivos impide que se sigan manteniendo estereotipos negativos frente a esta etapa de la vida y favorece el desarrollo de conocimientos especializados en el área, de gran utilidad y que evitan concebir al envejecimiento como sinónimo de enfermedad.

\section{Ese gran gerente general: nuestro cerebro}

El encéfalo consta de dos hemisferios: izquierdo y derecho, se le atribuyen distintas funciones a cada lado y su complementariedad hace que nuestras funciones sean integradas. El cerebro "frío y analítico" pareciera ser el izquierdo, mientras que el "bohemio e imaginativo" se atribuye al derecho. Se ha dicho que tiene un peso de alrededor $1.5 \mathrm{Kg}$, conformado por las células nerviosas denominadas: neuronas y neuroglias.

La capa externa del cerebro, o corteza cerebral, si se pudiese extender, sería , del tamaño de una servilleta con un grosor similar al de media docena de cartas de una baraja (Punset, 2006). Está organizada de una forma jerárquica entre las capas que la conforman, donde las áreas superiores se relacionan con la adaptación a nuevas situaciones y la predicción de eventos, mientras que las inferiores se asocian a actividades más básicas. 
En toda esta dinámica neuronal, existen componentes eléctricos y químicos que surgen en el contacto entre las células y el cual llaman el "beso nervioso", paso del impulso que se ha denominado: sinapsis. La liberación de sustancias o neurotransmisores ocurre gracias a las sinapsis químicas, a partir de vesículas que, de forma encapsulada, tienen la sustancia que requiere el organismo en determinado momento.

\section{El tiempo en el cerebro}

Calero y Navarro (2006) afirman que, hasta cierta edad, el funcionamiento cognitivo se encuentra estimulado por el ritmo de vida de cada persona, pero al llegar a la vejez y reducirse considerablemente las demandas del entorno, comienza un proceso de "desentrenamiento" que acaba siendo, al menos en parte, el responsable del declive que se produce en esta etapa de la vida.

Para Cohen (citado por Escobar, 2001) el envejecimiento cerebral puede definirse como la pérdida, aguda o crónica, de la capacidad de adaptación a cualquier cambio, necesaria para una vida de relación óptima.

Sería poco realista asumir que el envejecimiento está exento de cambios a nivel cerebral, entre ellos y lo más importante, es la muerte neuronal selectiva que ocurre en ciertas áreas corticales, así como la reducción en el tamaño de las neuronas. A partir de esto se explicaría lo que exponen Svennerholm et al. (citados por Álvarez, I et al., 2002) al afirmar que a los 100 años el cerebro pesa un $20 \%$ menos en mujeres y un $22 \%$ inferior en hombres.

Diversos trabajos en el área tienden a enfatizar la pérdida de neuronas en las áreas asociativas neocorticales y en la arquicorteza (hipocampo y giro dentado).

En general, los hombres pierden antes y en mayor cantidad tejido cerebral durante el envejecimiento, cuando se comparan con las mujeres. Ellos son más propensos a perder tejido en los lóbulos frontales y temporales, y las mujeres en el hipocampo y las áreas septales (Carter, citado por Duque-Parra, 2003). Además, el flujo sanguíneo encefálico se reduce en un $20 \%$.

Cada vez hay más indicios que apuntan al hecho de que las dos mitades del cerebro envejecen en forma diferente y los efectos de la atrofia que acarrea el envejecimiento del cerebro afectan al hemisferio derecho (encargado de las cosas nuevas) más rápidamente que al hemisferio izquierdo, vinculado a situaciones conocidas. Los 
sistemas neurotransmisores afectados son los acetilcolinérgicos de proyección cortical, es decir, acetilcolina; el sistema nigroestriatal dopaminérgico (dopamina) y los noradrenérgicos de proyección cortical (noradrenalina). Otros neurotransmisores parecieran no sufrir alteraciones significativas, como el glutamato, principal neurotransmisor excitatorio, relacionado con la capacidad de aprender.

Las alteraciones en el equilibrio entre noradrenalina y serotonina producen trastornos de ciclo vigilia-sueño, una de las principales quejas de las personas adultas mayores. Igualmente, la disminución noardrenérgica influye en los trastornos de memoria y sueño paradójico, así como en funciones asociadas al lóbulo frontal, provocando distractibilidad por estímulos irrelevantes (Román y Sánchez-Navarro, 1998).

Neuropsicológicamente, el envejecimiento cerebral normal se caracteriza por cambios cognitivos difusos poco discapacitantes, que son más acentuados en la esfera de la memoria, rapidez de pensamiento y razonamiento (Petersen et al., Zec, citados por García-Sánchez y Estéves-González, 2002).

\section{Buenas noticias para el cerebro envejecido}

Frente a los cambios en la cantidad de neuronas, se ponen en acción sistemas compensatorios a nivel bioquímico, a través de la producción de neurotransmisores por las neuronas indemnes y a nivel morfológico, con el aumento del árbol dendrítico de las neuronas intactas y la generación de nuevas neuronas.

El moderno estudio de la neuroplasticidad demuestra que los cerebros de las personas mayores no se degeneran, sino que tienen una evolución particular de acuerdo con la actividad realizada.

De esta forma se confirma la predicción de Hebb, de que el uso contribuye al mantenimiento de las sinapsis (Bauer, citado por Redolat y Carrasco, 1998), ya que la experiencia enriquecida parece producir cambios positivos en la neuroquímica y neuroanatomía cerebral, como el aumento del grosor cortical, de la ramificación neuronal o del número de sinapsis.

La neurogénesis en el cerebro adulto se evidencia en la formación de nuevas neuronas a nivel de bulbo olfatorio e hipocampo, lo que posee gran relevancia en la medida en que en casos donde se presenten muertes neuronales significativas sea posible inducir al encéfalo para que sustituya las neuronas perdidas (Kolb y Whishaw, 2006). 
Dentro de esa nueva perspectiva en las neurociencias, se consideraría que las neuronas y las células gliales se siguen reproduciendo durante toda la vida de los organismos, a nivel de bulbo olfatorio e hipocampo. Sin embargo, se desconoce la funcionalidad de esas nuevas neuronas que se forman, así como no es totalmente aceptada la regeneración a nivel de neocorteza.

El cerebro, por su capacidad plástica, va cambiando en respuesta a las modificaciones del entorno. Pueden variar las conexiones entre neuronas, modificar la red de capilares que les proporcionan oxígeno y nutrientes, y producir nuevas cadenas sinápticas en determinadas zonas cerebrales (Carvallo, citado por Valbuena., 2008).

\section{Desafiando los efectos del tiempo: cerebro trabajando}

Todos los teóricos de la ciencia gerontológica coinciden en que sentirse útil y tener un fuerte sentido de competencia personal asociada con la realización de actividades productivas y de corte intelectual que le sean interesantes, favorece positivamente al adulto mayor, tanto a nivel cortical como socio-emocional.

Por lo tanto, involucrarse en actividades voluntarias complejas promueve la generación de neuronas nuevas, así como modificaciones positivas en los procesos neurobiológicos, entre ellos, la activación neuronal, mejor circulación de la sangre en la zona cerebral y metabolismo de glucosa y oxígeno.

Si se analiza la naturaleza del cerebro, usualmente por ahorro de energía, no es proclive al cambio, de forma tal que la "higiene o entrenamiento mental" promueve cambios en la rutina diaria que le permiten, a la masa cerebral, aumentar los circuitos sinápticos y ampliar su rango de acción, reajustándose continuamente a las demandas del exterior (Carvallo, citado por Valbuena, 2008).

La creación de nuevas neuronas y el moldeaje cerebral puede incrementarse mediante el esfuerzo mental derivado de un entrenamiento cognitivo planificado y acorde con las necesidades de la persona adulta mayor, entendido como proceso de aprendizaje o reaprendizaje. Su ejecución dentro de este tipo de programas y sus respuestas a las actividades planteadas están íntimamente relacionadas con su grado educativo (cultural), el cual se ha demostrado es un importante factor protector de enfermedades neurodegenerativas, ya que se estimulan entramados sinápticos complejos, y frente a una alteración, existen interconexiones sinápticas alternas que se encargan de cumplir la función. Esto se ha denominado reserva 
cognitiva, y si bien no detiene algún proceso histopatológico que esté subyacente, sí influye en el grado que se expresen las alteraciones clínicas esperadas.

Factores como el aprendizaje, la práctica y la repetición constante son actividades que parecen estar asociadas no solo al desarrollo de nuevas neuronas, sino al mantenimiento de la salud, funcionalidad y prolongación del tiempo de vida (Instituto Nacional de Salud Mental, citado por Araya y Camargo, 2003).

Cada vez se acepta más la idea de que la educación otorga un efecto protector ante los trastornos cognitivos y resulta, además, un importante elemento predictor del vigor cognitivo en la adultez tardía. Pareciera no ser la educación en sí, sino la naturaleza de las actividades asociadas con la educación avanzada las que protegen contra la aparición de alteraciones cognitivas (Albert et al., citado por Goldberg, 2002).

Según Stern et al. (2004), durante el envejecimiento normal puede existir una reorganización neuronal de la reserva cognitiva, la cual forma parte de un proceso de compensación, frente a las consecuencias neurales del envejecimiento, que emplean en forma diferente las áreas cerebrales, si se compara con el desempeño de personas adultas jóvenes; la idea central de esta propuesta radica, entonces, en mantenerse activo y ocupado.

Queda claro, entonces, que la reserva cognitiva es algo maleable, cambiante con el curso de la vida y va a depender tanto de factores innatos como de las experiencias obtenidas, interrelacionados con otros factores como educación, puesto de trabajo desempeñado y las actividades de ocio a edad avanzada.

Las actividades de ocio también cumplen un rol fundamental en la preservación de las funciones cognitivas, ya que un estilo de vida caracterizado por entretenimientos de naturaleza social está asociado con un menor declive intelectual, y desarrollo de nuevas redes sinápticas más eficientes que proporcionan una reserva cognitiva que retrasa las manifestaciones clínicas de una patología neurodegenerativa.

Por tanto, en la manera que se utilice el cerebro durante toda la vida influirá sobre la cantidad de reserva cognitiva que se tenga.

\section{Hacia un envejecimiento activo}

Lainvestigación científicareciente sugiere unaimagen "bimodal" del envejecimiento, donde existen diferencias cruciales entre quienes pierden sus funciones intelectuales 
con la edad y quienes no lo hacen (succesfull aging o envejecimiento exitoso), por lo que en la actualidad se puede considerar un mito percibir la progresión a la vejez como un camino inexorable, predestinado y fatalista.

Entre los factores que juegan un papel destacado en el envejecimiento satisfactorio destacan el estado de salud, el ejercicio físico, la educación, nivel de inteligencia, personalidad, experiencias culturales, ocupación, entrenamiento cognitivo y el estilo de vida. Específicamente, los agentes relacionados con menor riesgo de declive cognitivo en la vejez tienen que ver con la participación en actividades que normalmente solo están disponibles en ambientes complejos e intelectualmente estimulantes (Schaie, citado por Redolat y Carrasco, 1998).

Hallazgos sobre neuroplasticidad, neurogénesis, calidad de vida y avances neuropsicológicos otorgan un abordaje amplio del envejecimiento, ya que las actividades que realiza la persona son de gran importancia, por lo que la vida mental intensa desempeña un papel esencial en el bienestar cognitivo en las etapas avanzadas de la vida e impacta en su percepción de autoeficiacia.

Cada vez más estudios sugieren que las capacidades innatas, medidas a través de un cociente intelectual a edad temprana, se ven influenciadas por las posteriores experiencias obtenidas y son ambos factores lo que contribuyen al grado de reserva que se puede tener cuando se llega a la vejez.

La integridad de las funciones cognitivas y la posibilidad de lograr un envejecimiento exitoso dependerá de la diversidad y características de las actividades que se manejen a lo largo de la vida, lo cual promoverá cambios estructurales y funcionales a nivel cerebral. Así que, los retos para el cerebro son fundamentales.

\section{Referencias}

Araya, L. y Camargo, F. (2003). Proliferación neuronal en humanos adultos: Una nueva esperanza terapéutica. Revista Vitae: Academia Biomédica Digital, 16.

Calero, M. y Navarro, E. (2006). Eficacia de un programa de entrenamiento en memoria en el mantenimiento cognitivo de ancianos con y sin deterioro cognitivo. Disponible en http://www.infocop.es/

Duque-Parra, J. E. (2003). Relaciones neurobiológicas y envejecimiento. Revista de Neurología, 36 (6), 549-554. Disponible en www.imsersomayores.csic.es/ documentos/boletin/2003/numero-02/articulo.pdf 
Escobar, A. (2001). Envejecimiento cerebral normal. Revista Mexicana de Neurociencias, 2(4), pp. 197-201.

García- Sánchez, C. y Estévez-González, A. (2002) Estimulación cognitiva en el envejecimiento y la demencia. Revista de Psiquiatría de la Facultad de Medicina, 29(6), 374-378. Disponible en www.nexusediciones.com/pdf/psiqui2002_6/ps-29-6-005.pdf

Álvarez, I., Barbosa, M., de Paz, F., Gil Verona, J., Macías, J.A., Maniega, M.A., Pastor, J. F. y Rami, L. (2002). Neuropsicología de la involución y el envejecimiento cerebral. Revista Española de Neuropsicología, 4(4). 262-282. Disponible en dialnet.unirioja.es/servlet/fichero_articulo?codigo=1006796\&orden $=72446$

Goldberg, E. (2002). El cerebro ejecutivo. Barcelona: Crítica.

Kolb y Whishaw (2006). Neuropsicología humana. Madrid: Editorial Médica Panamericana.

Redolat, R. y Carrasco, M. (1998). ¿Es la plasticidad cerebral un factor crítico en el tratamiento de las alteraciones cognitivas asociadas al envejecimiento? Revista Anales de Psicología, 14(1) 45-53. Disponible en www.um.es/analesps/ v14/v14_1/mv05v14-1.pdf

Román Lapuente, F. y Sánchez Navarro, J. (1998). Cambios neuropsicológicos asociados al envejecimiento normal. Revista Anales de Psicología, 14(1), $27-$ 43. Disponible en www.um.es/facpsi/analesps/v14_1e.htm - 34k

Punset, E. (2006). El alma está en el cerebro. Madrid: Punto de Lectura.

Stern, Y., Scarmeas, N. y Habeck, C. (2004). Imaging cognitive reserve. International Journal of psychology, 39(1), 18-26. Disponible en www.cumc.columbia.edu/dept/sergievsky/cnd/pdfs/ImagingCognitiveReserve2.pdf

Valbuena, M. (2008). Una mente saludable (Entrevista realizada al Dr. E. Carva1lo). Revista Estampas. Disponible en www.eluniversal.com/estampas/anteriores/141007/salud.shtml 